\title{
An Advanced Emergency Warning Message Scheme based on Vehicles Speed and Traffic Densities
}

\author{
Mustafa Banikhalaf $^{1}$, Saleh Ali Alomari ${ }^{2}$, Mowafaq Salem Alzboon ${ }^{3}$ \\ Faculty of Information Technology and Computer science, Yarmouk University, Jordan, Irbid $^{1}$ \\ Faculty of Science and Information Technology, Jadara University, Jordan, Irbid ${ }^{2}{ }^{3}$
}

\begin{abstract}
In intelligent transportation systems, broadcasting Warning Messages (WMs) by Vehicular Ad hoc Networks (VANETs) communication is a significant task. Designing efficient dissemination schemes for fast and reliable delivery of WMs is still an open research question. In this paper, we propose a novel messaging scheme, Advanced Speed and Density Warning Message (ASDWM). ASDWM is a broadcast-based scheme that meets design objectives and achieves high saved rebroadcast and reachability, as well as low end-to-end latency of WM delivery. The ASDWM uses vehicle speeds and vehicles density degrees to help emergency vehicles to send WM according to a road condition, adaptively. Simulation results demonstrate the effectiveness and superiority of the ASDWM over its counterparts.
\end{abstract}

Keywords-Warning message; the broadcast storm problem; emergency vehicles

\section{INTRODUCTION}

Inside a city or on the highway, unexpected events such as traffic accidents and medical emergencies occur every day. Hence, it is very critical for emergency vehicles to reach an accident spot as soon as possible. In case of such unexpected events, an emergency vehicle should inform other vehicles and traffic lights ahead to clear the way for it. Basically, all emergency vehicles are equipped with a wireless card to detect the event and utilize the underlying VANET architecture to issue WMs. One of the most important challenges in this scenario is to design a warning message dissemination scheme to transfer the WM in a reliable and low-latency broadcasting. It is crucial that all vehicles and traffic lights in front of emergency vehicles receive the WM with high probability, and with the minimum possible delay to take a proper action very quickly.

A simple solution to the above problem is handled using blind flooding [1]. It is a primitive scheme that allows each vehicle to rebroadcast the WM when it receives it for the first time to all surrounding vehicles exactly once. Blind flooding trends to be an optimal solution in low sparse networks. However, using it in cases of high vehicular traffic densities cause several communication channel problems. Network performance drops down due to a considerable number of duplicated generated messages. These duplicated messages can cause message collisions, increase occupancy or contention on available channel capacity, which leads to high latency in delivering WM. This problem is widely known as the broadcast storm problem [1], and several solutions have been proposed in the literature to mitigate its effects [2][3][4]. The main idea of these solutions has been to limit rebroadcasting the message to candidate vehicles and to guarantee that all other vehicles received it. Each proposed solution uses a different method to choose group of vehicles that are responsible to forward WM with minimum overhead, latency and high reachability.

In VANETs, the most effective and reliable broadcast approach is to privilege a broadcast operation to any vehicle that located on the transmission range of the source vehicle [5][6]. However, this approach cannot be operated without Global Positioning System (GPS) availability. It is commonly known that the strength of a GPS signal is often influenced by external environment conditions, which imposes several issues when using this approach. Other proposed approaches use a WM prioritization technique [7] to make GPS based schemes more efficient. It is assumed that the WM should be assigned a high priority at MAC layer and broadcasted before other types of messages. Although this technique helps some safety applications to guarantee that the WM is delivered as fast as possible, but time delay to broadcast two consecutive WMs depends on a fixed slot time. This increases number of unnecessary WMs, which reduces network communication efficiency. The main contribution in this work is to: (i) develop (ASDWM) scheme, (ii) and to adjust the required time delay before broadcasting the next WM dynamically, (iii) which eventually mitigates side effect of the broadcast storm problem.

The rest of the paper is organized as follows. Section II reviews the related work on the probabilistic broadcasting schemes. Section III presents ASDWM. Section IV shows the simulation environment used to validate the ASDWM, presents and discusses the obtained results. Finally, Section V concludes this paper.

\section{RELATED WORKS}

Over the last decades, probabilistic broadcasting schemes have been implemented using several techniques such as probabilistic density-based schemes [3]-[8], probabilistic counter-based schemes [9][10] and [11], probabilistic GPSbased schemes [12][13][14], and probabilistic algebraic-based scheme [15]. Several studies show that probabilistic schemes that depends on a GPS device are the appropriate solutions for several communication scenarios in VANETs. However, GPS availability and reliability are not always guaranteed, which makes these studies not always applicable. In this paper, we shed the light on some important probabilistic schemes which require GPS availability, followed by the most effective and relevant studies that depend on the vehicles speed as a 
parameter to calculate the rebroadcast probability [16] and [17].

\section{A. Probabilistic GPS based-Schemes}

Irresponsible Forwarding (IF) [12], is a broadcasting scheme presented for VANETs which combines advantages of both distance and density schemes to calculate a dynamic forwarding probability value. The inter-vehicle spacing distribution in the network, and the distance between two vehicles are used to calculate the forwarding probability using the following expression:

$\mathrm{p}=\left(1-\mathrm{F}_{\mathrm{x}}(\mathrm{z}-\mathrm{d})\right)^{1 / \mathrm{k}}$

where $\mathrm{X}$ denotes the distance between consecutive vehicles, $d$ is a distance between a transmitter and a receiver, and $\mathrm{z}$ is the transmission range, $\left.F_{x}(z-d)\right)$ is the Cumulative Distributed Function (CDF), and $\mathrm{k}$ is a shaping parameter used to adjust the rebroadcast probability. Authors in [18] have proposed CAREFOR scheme based on IF. It assumes that is not practical for each vehicle in the network has the same transmission range. Hence, CAREFOR includes ratio accounts for difference in the vehicle's transmission range.

Weighted P-Persistence (WP-P) [19] is another well-known scheme that uses the distance value between vehicles as an input to (2), to determine the forwarding probability, where the rebroadcast operation is privileged to farthest vehicles always:

$P_{i j}=\frac{\text { Dist }_{i j}}{T_{r}}$

where Dist $_{i j}$ is the distance between vehicle $\mathrm{i}$ and vehicle $\mathrm{j}$, and $T_{r}$ is the transmission radio range. Several similar GPS based schemes have been proposed in [20] and [21], which use vehicles coordination positions to calculate the rebroadcast value carefully. Interested readers can refer to these studies to gain deep knowledge and understanding.

\section{B. Probabilistic Speed-based Schemes}

Speed Adaptive Broadcast (SAB) is proposed in [16] to adaptively adjust the forwarding probability based on the speed of traveling vehicle. A ratio between the speed value of current vehicle $i$ at time $t$, and the limit of speed allowed $V_{x}$ for road vehicles, are used by the following equation to calculate the forwarding probability:

$P(i, t)=\frac{v(i, t)}{V_{x}}$

In [17], the authors proposed Speed Adaptive Probabilistic Flooding (SAPF) to estimate traffic densities in VANETs based on the vehicle's speed, and to adjust the forwarding probability according to the following equation:

$\mathrm{P}=0.055 \mathrm{v}-0.033$

where $\mathrm{v}$ is the vehicle's speed. SAPF defines two types of speed thresholds are vl and vh which represents vehicles that move with low speed, and vehicles that move with high speed respectively. Estimating the vehicle's density when $v>$ vh is impossible. Hence, the direct blind flooding is used instead to guarantee high message reachability. On the other hand, if $\mathrm{v}<$ $\mathrm{vl}$, a fixed value of the forwarding probability is used as the maximum network capacity has been reached.

\section{ASDWM DESCRIPTION}

The significant feature of the ASDWM is to regulate the rebroadcast probability according to traffic densities and the vehicle speed. The main aim is to guarantee that WM is reached all vehicles with low latency for a period of time. Vehicles move with low speeds inside a city indicate high vehicle density due to traffic jam, accidents or other potential hazards. In this case, it is sufficient to use a low forwarding probability value to disseminate the WM to all vehicles with minimum cost. In the other hand, when vehicle speeds inside a city reach the maximum speeds this implies low vehicular densities, and high rebroadcast probability values are required to achieve a high percentage of the WM delivery. Therefore, the ASDWM categorizes traffic densities based on the vehicle speed into three regimes; low-density, medium-density and high-density. The low-density regime means that the speed of vehicle reaches to the maximum speed and the rebroadcast probability is set to be equal to 1 (i.e., flooding). We use the flooding technique in this regime to guarantee message delivery to all vehicles. Normally, inside the medium-density regime a vehicle travels at a speed above $10 \mathrm{~km} / \mathrm{h}$ and below the maximum speed. Hence, the rebroadcast probability is set to be equal 0.7 to balance between high message reachability and low retransmission [3]. In the high-density regime, when the vehicle moves with a speed less than $10 \mathrm{~km} / \mathrm{h}$, it is not practical to set a fixed value for the rebroadcast probability. Therefore, the following strategy is used to estimate the accurate density level and adjust the rebroadcast probability accordingly. Three density levels (density level 1, density level 2 and density level 3) are calculated based on neighboring information to calculate the best of value the rebroadcast probability.

Density Level 1 (DL1) represents a density measurement of a set of 1-hop neighborhood information that can be covered via a set of 2-hop neighborhood information. Assume that $S_{v}^{1}$ is a set of 1-hop neighbors of a vehicle v, and $S_{v}^{2}$ is a set of 2-hop neighbors of $\mathrm{v}$. $\mathrm{N}$ denotes the number of elements in each set. DL1 is calculated as follows:

$\mathrm{DL} 1=\frac{\mathrm{N}\left(\mathrm{S}_{\mathrm{V}}^{1}\right)}{\mathrm{N}\left(\mathrm{S}_{\mathrm{V}}^{1}\right)+\mathrm{N}\left(\mathrm{S}_{\mathrm{V}}^{2}\right)}$

Density Level 2 (DL2) represents a density measurement of a set of 2-hop neighborhood information within 2-hop neighborhood information. DL2 is calculated as follows:

$\mathrm{DL}=\frac{\mathrm{N}\left(\mathrm{S}_{\mathrm{V}}^{2}\right)}{\mathrm{N}\left(\mathrm{S}_{\mathrm{V}}^{1}\right)+\mathrm{N}\left(\mathrm{S}_{\mathrm{V}}^{2}\right)}$

Density Level 3 (DL3) represents a density measurement of a set of 2-hop neighborhood information that can only be reached via a 1-hop neighbor. Assume that $S_{v, x_{k}}^{3}$ is the set of 2hop neighborhood information that can only be reached via 1hop neighborhood information $x_{k}$ from a given vehicle $\mathrm{v}$ (for $\mathrm{k}$ $=1,2,3, \ldots, n)$. DL3 is calculated as follows:

$\mathrm{DL} 3=\frac{1}{\mathrm{~N}\left(\mathrm{~S}_{\mathrm{v}}^{1}\right)} \sum_{\mathrm{k}=1}^{\mathrm{N}\left(\mathrm{S}_{\mathrm{v}}^{1}\right)} \mathrm{N}\left(\mathrm{S}_{\mathrm{v}, \mathrm{x}_{\mathrm{k}}}^{3}\right)$

Based on the collected information from DL1, DL2 and DL3, the rebroadcast probability value that to be used in the high-density regime is calculated as follows: 
$\mathrm{P}=\frac{\mathrm{DL} 1+\mathrm{DL} 2+\mathrm{DL} 3}{3}$

The WM must be queued with the high priority at MAC layer. Enhanced Distributed Channel Access (EDCA) is the fundamental channel access mechanism of Wireless Access for Vehicular Environment (WAVE). It offers four Access Classes (ACs) to priorities messages based on their types and applications. ACO indicates that a broadcast message has the lowest priority, and AC3 it has the highest priority [22]. The WM in this work is assigned AC3 to make sure it is delivered before any other types of messages.

The most related studies [7] and [23] use fixed delay time between broadcasting two consecutive WMs. In fact, this implementation is not practical in real life scenarios, and makes the broadcast storm problem worse. the ASDWM adopts the same density principle to adjust time delay when broadcasting WMs. The following equation is used:

Time_Delay $=\left(\frac{\mathrm{DL} 1+\mathrm{DL} 2+\mathrm{DL} 3}{3}\right) \times \Delta(\mathrm{t}) \max$

where $\Delta(t) \max$ is a small random waiting time. Using the delay time (9) can regulate the period between consecutive broadcasting message adaptively. For instance, when a vehicle moves inside the high-density regime, delay time is preferable to be long as one broadcast is enough to reach all vehicles. While in the low-density regime delay time should be too short to keep sending the WM until arrives a destination area. The ASDWM steps are organized in Fig. 1.

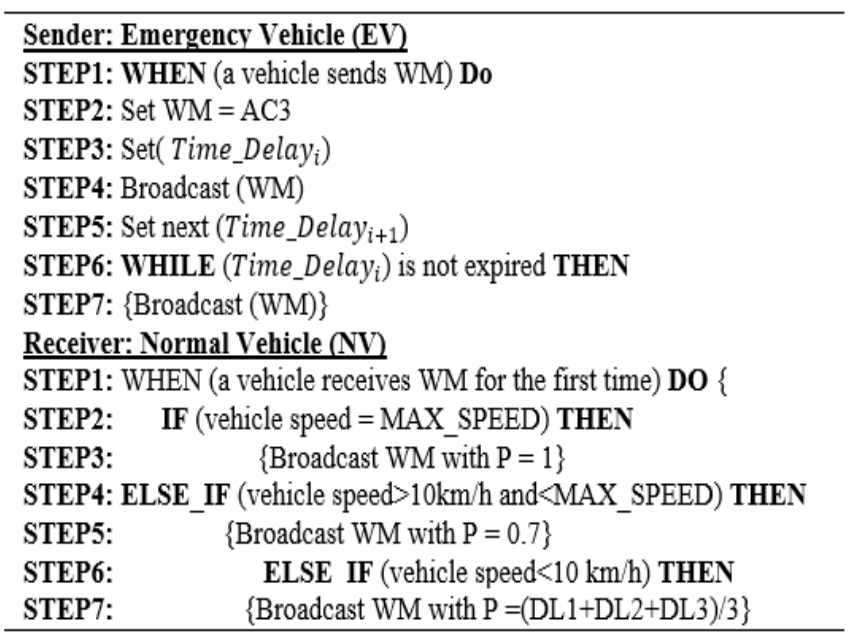

Fig 1. The ASDWM Scheme Logical Steps.

\section{Example on ASDWM}

In Fig. 2 scenario (a), the emergency vehicle travels with $60 \mathrm{~km} / \mathrm{h}$, which is the maximum speed limit on the road. Normally, when the driver notices that the road is empty, he accelerates the vehicle speed until it reaches the maximum speed limit. For this reason, the emergency vehicle keeps sending the WM every short regular period based on the density input to (9). As DL1, DL2 and DL3 is equal to 0. In Fig. 2 scenario (a), Time_Delay is set to $10^{-3} \mathrm{~ms}$. Once the vehicle $A$ receives the $\mathrm{WM}$ from the emergency vehicle, it retransmits it with probability $\mathrm{p}=1$, as no vehicle in the neighborhood rebroadcasts to prevent the WM's dying out.

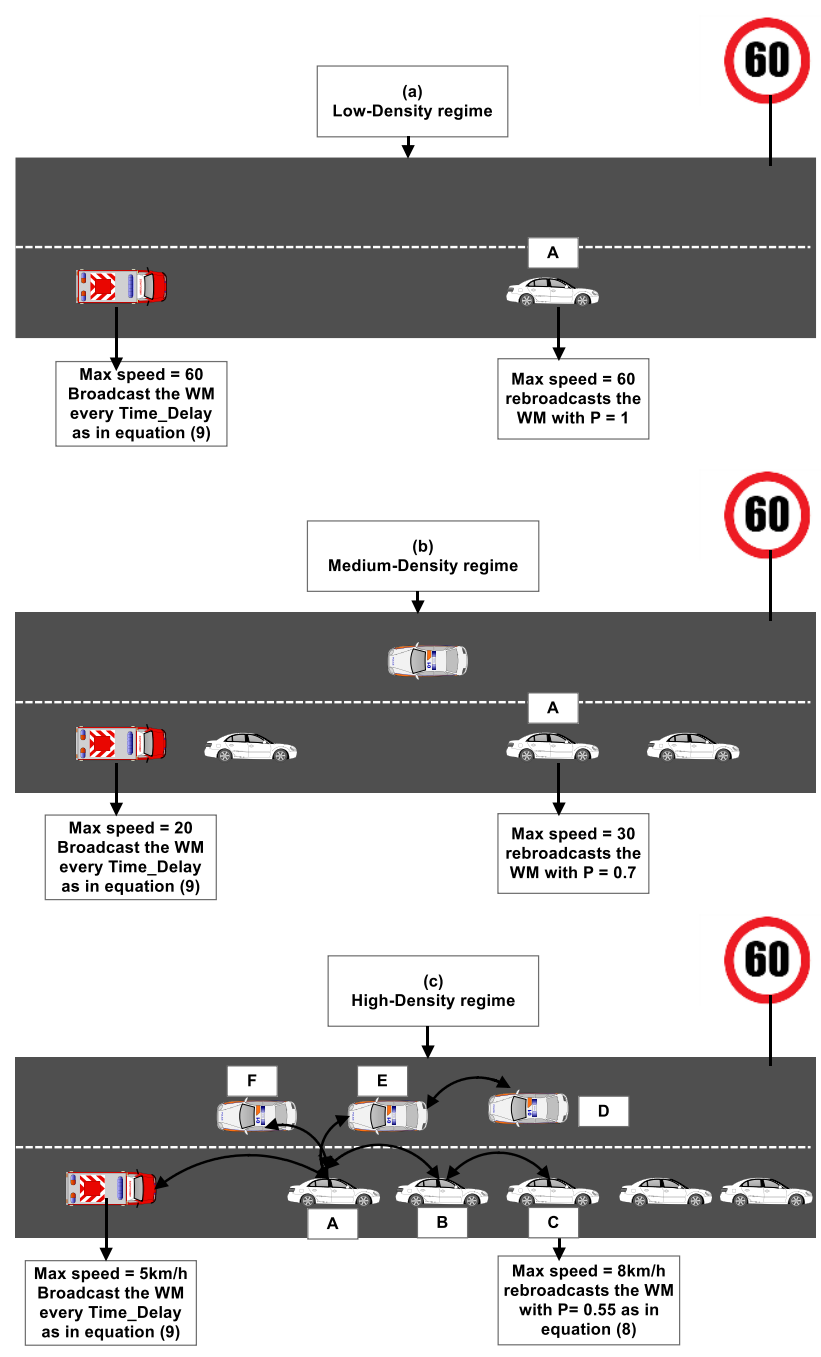

Fig 2. Adjusting the rebroadcast probability value according to the traffic densities.

In the medium-density regime as in Fig. 2 scenario (b), small probability value may lead to poor message reachability, and high probability value leads to the broadcast storm problem.

Hence, the rebroadcasting probability should be set to at least 0.7 to balance between message reachability and low retransmission [3]. Time_delay between consecutives WMs is also calculated based on (9).

The serious side effect of the broadcast storm problem often appears in the high-density regime as shown in Fig. 2 scenario (c). Hence, the rebroadcasting probability must be chosen carefully to mitigate its side effect as much as possible. Usually, vehicles are considered inside the high-density regime if it travels with speed less than $10 \mathrm{~km} / \mathrm{h}$. In this case, the rebroadcasting probability is set dynamically with respect to different density levels that are calculated in (8). For instance, when the vehicle A receives the WM from the emergency vehicle, it calculates DL1, DL2, DL3, P and Time_Delay as follows: 


$$
\begin{aligned}
& \mathrm{DL} 1=\frac{\mathrm{N}\left(\mathrm{S}_{\mathrm{v}}^{1}\right)}{\mathrm{N}\left(\mathrm{S}_{\mathrm{v}}^{1}\right)+\mathrm{N}\left(\mathrm{S}_{\mathrm{v}}^{2}\right)}=\frac{3}{3+2}=0.6 \\
& \mathrm{DL} 2=\frac{\mathrm{N}\left(\mathrm{S}_{\mathrm{v}}^{2}\right)}{\mathrm{N}\left(\mathrm{S}_{\mathrm{v}}^{1}\right)+\mathrm{N}\left(\mathrm{S}_{\mathrm{V}}^{2}\right)}=\frac{2}{3+2}=\frac{2}{5}=0.4 \\
& \begin{array}{r}
\mathrm{DL} 3=\frac{1}{\mathrm{~N}\left(\mathrm{~S}_{\mathrm{v}}^{1}\right)} \sum_{\mathrm{k}=1}^{\mathrm{N}\left(\mathrm{S}_{\mathrm{V}}^{1}\right)} \mathrm{N}\left(\mathrm{S}_{\mathrm{v}, \mathrm{x}_{\mathrm{k}}}^{3}\right)=\frac{1}{3} \sum_{\mathrm{k}=1}^{3} 1+1+0=\frac{2}{3}=0.66 \\
\mathrm{P}=\frac{\mathrm{DL} 1+\mathrm{DL} 2+\mathrm{DL} 3}{3}=\frac{0.6+0.4+0.66}{3}=0.55 \\
\quad=\left(\frac{0.6+0.4+0.66}{3}\right) \times 10^{-3} \\
=0.55 \times 10^{-3}
\end{array}
\end{aligned}
$$

where $N\left(S_{v}^{1}\right)$ and $N\left(S_{v}^{2}\right)$ are set of 1-hop neighbors [ B, E, $\mathrm{F}]=[3]$, and set of 2-hop neighbors $[\mathrm{D}, \mathrm{C}]=[2]$ of the vehicle A, respectively. Set of 2-hops neighbors that can only be reached via 1-hop neighbor $N\left(S_{v, x_{k}}^{3}\right)$ for the vehicle $\mathrm{A}$ is collected as follows: $S_{A, B}^{3}=[\mathrm{C}]=[1], S_{A, E}^{3}=[\mathrm{D}]=[1]$ and $S_{A, F}^{3}=$ $[0]$.

\section{Simulation SETUP AND RESUlt ANALYSIS}

The performance of ASDWM is evaluated through simulation using NS-2 simulation environment [24], and it is compared against the most related scheme SAPF [17]. Traffic flows are generated using SUMO [25]. The physical layer frequency is adjusted to $5.9 \mathrm{GHz}$ according to DSRC standard, and Bandwidth is set to $10 \mathrm{MHz}$ [26]. The transmission range communicating value is adjust to 250 meters. Between 100 and 500 vehicles are uniformly distributed on a road consisting of two lanes $5 \mathrm{Km}$ length that is similar to Fig. 2. Vehicles travel at different speed between minimum $0 \mathrm{~km} / \mathrm{h}$ up to $\max 60 \mathrm{~km} / \mathrm{h}$ throughout the lanes. The following metrices are used to observe the network performance:

- Reachability: It is measured by the percentage of vehicles receiving the WM, divided by the total number of vehicles that are reachable.

- Saved Rebroadcast: It shows the ratio between the numbers of vehicles receiving the WM and the number of vehicles rebroadcasting it.

- Latency: It is the time between sending the WM from the source, until the time it reached the destination.

Fig. 3 illustrates the reachability achieved by Flooding, SAPF and ASDWM when the number of vehicles is varied. Basically, reachability always increases with increased number of vehicles. This is because when the density of vehicle increases, the distance between neighbors decreased and the number of vehicles covering a road segment increase. For instance, reachability achieved by Flooding increases from $70 \%$ for 50 vehicles to $100 \%$ for 200 vehicles, while the reachability achieved by SAPF and ASDWM increases from $30 \%$ to $100 \%$ for 30 and 200 vehicles, respectively. As expected, Flooding achieves the best reachability performance compared to the other schemes, as it allows all vehicle to rebroadcast the WM. On the other hand, the reachability performance results in the dense network for SAPF and ASDWM are similar and comparable to Flooding.

Fig. 4 shows that the latency incurred by Flooding, SAPF and ASDWM increases with increased network density. All the schemes have similar latency when number of vehicles is equal 100. After this point, Flooding incurs the highest latency compared to other schemes. It is noticed from the Fig. 4 that ASDWM incurred the lowest latency compared to the Flooding and SAPF in the sparse and the dense network. This is because the ASDWM adjusts the Time_Delay between WMs according to traffic densities, which reduces the number of retransmissions, prevents contentions and message collisions in the vehicular networks. Fig. 5 shows the saved rebroadcast reported by Flooding, SAPF and ASDWM as network density increased. Normally, the vehicular network becomes denser as the number of vehicles increased, which makes all the schemes to rebroadcast a larger number of unnecessary WMs. it shows that the saved rebroadcast for Flooding scheme is equal to zero as all vehicles are eligible to retransmit the received WM. The figure also shows that as the network densities increased, the ASDWM achieves significant percentage of the saved rebroadcast compared to the SAPF. For instance, compared with the SAPF, the saved rebroadcast in the ASDWM can increase further by approximately $20 \%$ when the number of vehicles is relatively large (e.g., 500 vehicles).

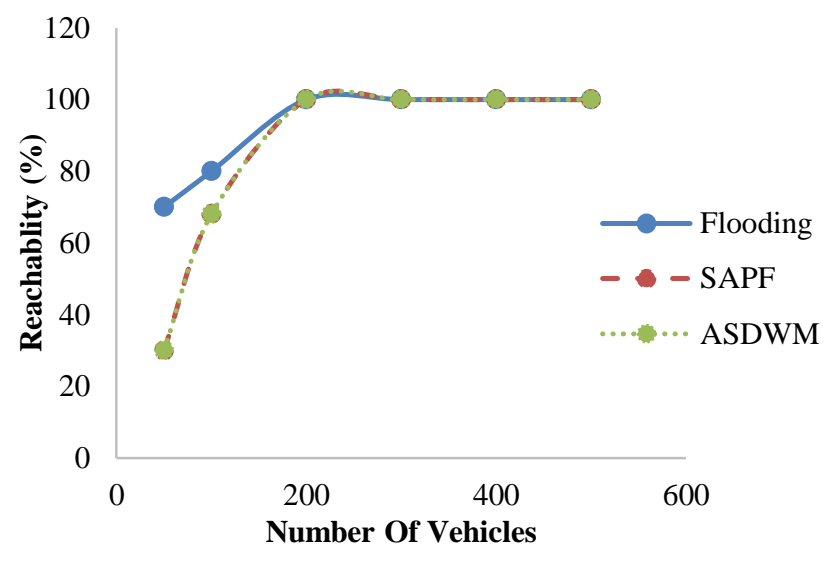

Fig 3. Reachability (\%) vs Number of Vehicles.

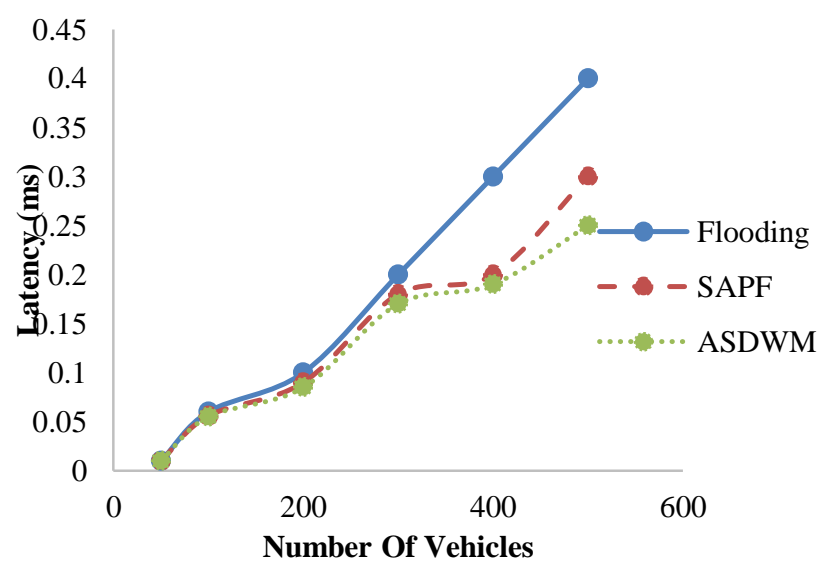

Fig 4. Latency (ms) vs Number of Vehicles. 


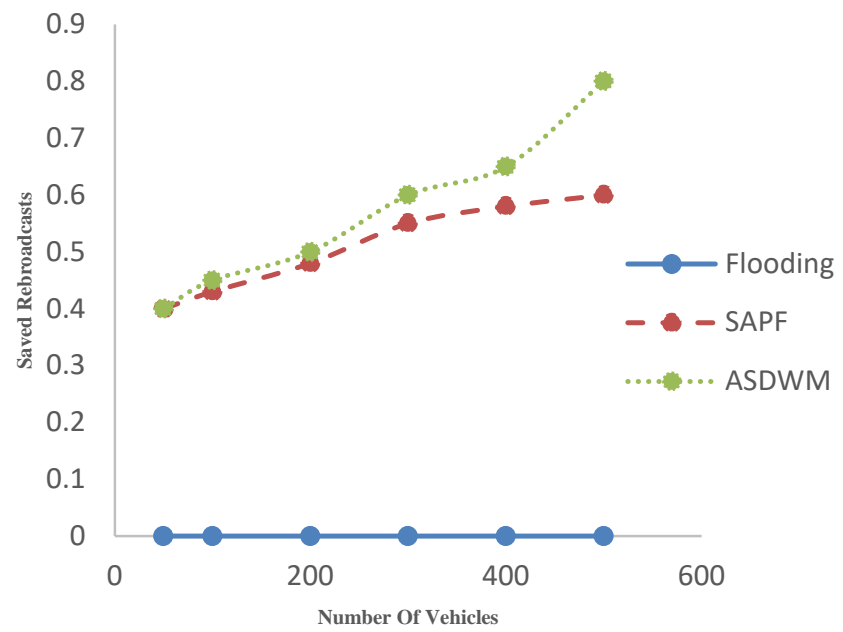

Fig 5. Saved Rebroadcasts vs Number of Vehicles.

\section{CONCLUSION AND FUTURE WORKS}

In this paper, we presented ASDWM, which is designed to disseminate WMs over VANETs in urban environments. The WMs can be quickly and reliably delivered by the emergency vehicles to the target destination with lowest latency and highest saved rebroadcast, without scarifying reachability. Simulation results show that in comparison with Flooding and SAPF protocols, ASDWM achieves the minimal message delivery latency, and keeps reachability is equivalent to Flooding in high densities area. Moreover, ASDWM can maintain more than $30 \%$ and $100 \%$ saved rebroadcast compared to SAPF and Flooding, respectively. For the future works, ASDWM can be extended further to include vehicle's direction when broadcasting WMs. This makes it more applicable with real life scenarios and VANETs applications.

\section{REFERENCES}

[1] Y.-C. Tseng, S.-Y. Ni, Y.-S. Chen and J.-P. Sheu," The broadcast storm problem in a mobile ad hoc network," Wireless Networks, vol.8, no.2-3, pp.153-167, Mar.2002.

[2] M. B.Yassein, M. B. Khalaf and A. Y. Al-Dubai," A new probabilistic broadcasting scheme for mobile ad hoc on-demand distance vector ( AODV ) routed" The Journal of Supercomputing,vol.53, no.1,pp.196$211,2010$.

[3] Z. J. Haas, J. Y. Halpern and L. Li, "Gossip-based ad hoc routing," IEEE/ACM Transactions on Networking, vol. 14, no. 3, pp. 479-491, June.2006.

[4] J. Cartigny and D. Simplot," Border node retransmission based probabilistic broadcast protocols in ad-Hoc networks," Telecommunication Systems, vol. 22, pp.189-204, 2003.

[5] D. G. Reina, S. L. Toral, P. Johnson and F. Barrero "Improving discovery phase of reactive ad hoc routing protocols using Jaccard distance," The Journal of Supercomputing, vol.67, pp 131-152, January 2014.

[6] G. Korkmaz, E. Ekici and F. Ozguner, "An efficient fully ad-hoc multihop broadcast protocol for inter-vehicular communication systems," IEEE International Conference on Communications (ICC 2006) , Istanbul, Turkey, pp. 423-428.

[7] F. J. Martinez, C.-K. Toh, J.-C. Cano, C. T. Calafate, P.Manzoni, "A Street Broadcast Reduction Scheme (SBR) to Mitigate the Broadcast Storm Problem in VANETs," Wireless Personal Communications, vol.56, pp. 559-572, Feb. 2011.
[8] A. Y. Al-Dubai, M. B. Khalaf, W. Gharibi and J. Ouenniche, "A new adaptive probabilistic broadcast protocol for vehicular networks," IEEE 81st Vehicular Technology Conference (VTC 2015), Glasgow, pp. 1-5.

[9] M. Chekhar, K. Zine-Dine, M. Bakhouya and A. Aaroud, "A dynamic threshold-based probabilistic scheme for broadcasting in ad hoc networks," 15th International Conference on Intelligent Systems Design and Applications (ISDA 2015), Marrakech, pp. 511-516.

[10] J. Sospeter, D. Wu, S. Hussain, and T. Tesfa, "An Effective and Efficient Adaptive Probability Data Dissemination Protocol in VANET," Data, vol. 4, no. 1, p. 1, 2018.

[11] A. Mohammed, M. Ould-Khaoua, L. M. Mackenzie and J. Abdulai, "Dynamic probabilistic counter-based broadcasting in mobile ad hoc networks," 2nd International Conference on Adaptive Science \& Technology (ICAST 2009), Accra, pp. 120-127.

[12] S. Panichpapiboon and L. Cheng, "Irresponsible forwarding under real intervehicle spacing distributions," IEEE Transactions on Vehicular Technology, vol. 62, no. 5, pp. 2264-2272, Jun.2013.

[13] M. B. Khalaf , A. Y. Al-Dubaia and G. Minb, "New efficient velocityaware probabilistic route discovery schemes for high mobility Ad hoc networks", Journal of Computer and System Sciences,vol.81, pp.97-109, Feb.2015.

[14] W. Wang, T. Luo, H. Kang, and M. Elhoseny, "A local information sensing-based broadcast scheme for disseminating emergency safety messages in IoV," Mob. Inf. Syst., vol. 2019, 2019.

[15] G. Koufoudakis, K. Oikonomou, K. Giannakis, and S. Aïssa, "Probabilistic flooding coverage analysis for efficient information dissemination in wireless networks," Comput. Networks, vol. 140, pp. $51-61,2018$.

[16] M. Chaqfeh and A. Lakas, "Speed adaptive probabilistic broadcast for scalable data dissemination in Vehicular Ad Hoc Networks," International Wireless Communications and Mobile Computing Conference (IWCMC 2014), Nicosia, pp. 207-212.

[17] Y. Mylonas, M. Lestas, A. Pitsillides and P. Ioannou, "Speed Adaptive Probabilistic Flooding for vehicular ad-hoc networks," IEEE 22nd International Symposium on Personal, Indoor and Mobile Radio Communications (PIMRC.2011), Toronto, 2011, pp. 719-723.

[18] A. Mostafa, A. M. Vegni, and D. P. Agrawal, "A probabilistic routing by using multi-hop retransmission forecast with packet collision-aware constraints in vehicular networks," Ad Hoc Networks, vol.14, pp.118$1129,2014$.

[19] N. Wisitpongphan, et al., "Broadcast storm mitigation techniques in vehicular ad hoc networks," IEEE Wireless Communications, vol. 14, no. 6, pp. 84-94, Dec. 2007.

[20] B. Yuan, A. jie and Z. Huibing,"Location aided probabilistic broadcast algorithm for mobile Ad-hoc network routing," The Journal of China Universities of Posts and Telecommunications, vol.24, pp. 66-71, 2017.

[21] I. A. Khan, A. Javaid and H. L. Qian, "Distance-based dynamically adjusted probabilistic forwarding for wireless mobile Ad Hoc Networks," 5th IFIP International Conference on Wireless and Optical Communications Networks (WOCN 2008), Surabaya, pp. 1-6.

[22] S. Eichler, "Performance evaluation of the IEEE 802.11p WAVE communication standard," 66th IEEE Vehicular Technology Conference (VTC 2007), Baltimore, MD, pp.2199-2203.

[23] F. J. Martinez, et al., "Evaluating the impact of a novel warning message dissemination scheme for VANETs using real city maps", 9th International IFIP- TC6 Networking Conference, Chennai, India, pp.265276, May. 2010.

[24] McCanne, S., Floyd, S.: The Network Simulator - ns-2.

[25] D. Krajzewicz, G. Hertkorn, C. Rossel, and P. Wagner, "SUMO (Simulation of Urban MObility)-An open-source traffic simulation," Proceedings of the 4th Middle East Symposium on Simulation and Modelling (MESM 2002), Sharjah, UAE, pp.183-187.

[26] Standard Specification for Telecommunications and Information Exchange between Roadside and Vehicle Systems, _5 $5 \mathrm{GHz}$ Band Dedicated Short Range Comm. (DSRC) Medium Access Control (MAC) and Physical Layer (PHY) Specifications, ASTM E2213-03, Sept. 2003. 